doi: $10.2306 /$ scienceasia1513-1874.2013.39.319

\title{
When a probability interval is a random set
}

\author{
Peeraporn Boodgumarn ${ }^{\mathrm{a}}$, Phantipa Thipwiwatpotjana ${ }^{\mathrm{a}, *}{ }^{\mathrm{w}}$, Weldon A. Lodwick \\ ${ }^{a}$ Department of Mathematics and Computer Science, Faculty of Science, Chulalongkorn University, \\ Bangkok, Thailand \\ b Department of Applied Mathematical and Statistical Sciences, University of Colorado at Denver, Colorado, \\ USA
}

${ }^{*}$ Corresponding author, e-mail: phantipa.t@ chula.ac.th

Received 6 Nov 2012

Accepted 10 May 2013

\begin{abstract}
Some uncertainties can be represented by random sets, while some others may be in the form of probability intervals. Even though random sets and probability intervals are defined differently, we may be able to represent a given probability interval as a unique random set when this probability interval satisfies some conditions. In this paper, we present these conditions which will check whether or not there is a unique random set expressing the same information as a given probability interval. We construct the random set when the conditions are satisfied. We also give examples of when a user should use a random set over a given probability interval.
\end{abstract}

KEYWORDS: basic probability assignment function, reachable probability interval

\section{INTRODUCTION}

Probability intervals ${ }^{1}$ and random sets $^{2-4}$ are two examples of many interpretations of uncertainty ${ }^{5-9}$. Probability intervals are easy to present and users can readily understand them. However, a user may not want to interpret their probability interval information when they know that the probability interval provides the same information as a random set. Hence the user may wish to use the random set to represent the data. For example, instead of telling others that a loaded die has a probability interval (based on statistical experiments) information, it is perhaps better for a casino to provide a random set version of this information.

The definitions of a probability interval and a random set are not equivalent. Shafer ${ }^{4}$ proved that a belief measure associated with a random set has an $\infty$ monotone capacity, while De Campos et al ${ }^{1}$ proved that a reachable probability interval can be expressed using its tight bounds on probabilities as Choquet capacity of order 2. Lemmer and Kyburg ${ }^{10}$ developed an inner approximate transformation of a set $\mathcal{P}_{L}$ of probabilities generated by a reachable probability interval into a set $\mathcal{P}_{\mathrm{Bel}}$ of probabilities that was generated by a random set. In addition to an inner approximation, Deneoeux ${ }^{11}$ studied the transformation of a set $\mathcal{P}_{L}$ into a set of probabilities generated by a random set using an outer approximation. Thipwiwatpotjana and Lodwick $^{12,13}$ provided a construction of a random set for a given probability interval that is easier to apply than the existence construction in Ref. 10. However, there has been no attempt to find conditions for which these two sets are equal. Hence if we can find the conditions for which $\mathcal{P}_{L}=\mathcal{P}_{\text {Bel }}$, it means that a reachable probability interval with these conditions can extend its Choquet capacity to be $\infty$-monotone.

This paper develops the conditions for which a given probability interval represents the same information as that of a unique random set enabling us to extend the 2-monotone capacity of this probability interval to the $\infty$-monotone capacity. Furthermore, if we have such information, we could use either a probability interval or a random set to represent this information. The paper is divided as follows. We provide the definitions of a probability interval and a random set in the next section. Then we present conditions for which a probability interval is a random set. These conditions lead to the construction of the associated random set given in Theorem 1. Finally, an example and conclusion of this paper are provided.

\section{DEFINITIONS AND NOTATION}

We first provide definitions of a probability interval, a random set, and other related terms needed in this paper. The scope of our work is limited to the finite case of uncertainty information realizations.

Definition 1 (see Section 2 of Ref. 5) Given $X=$ $\left\{x_{1}, x_{2}, \ldots, x_{n}\right\}$ as the set of realizations of uncertainty information, a capacity on $X$ is a function $\mu$, defined on the set of subsets of $X$, such that: (i) $\mu(\varnothing)=0, \mu(X)=1$; (ii) $A \subseteq B \longrightarrow \mu(A) \leqslant$ 
$\mu(B)$. A capacity such that

$$
\mu(A \cup B) \geqslant \mu(A)+\mu(B), \forall A, B \subseteq X,
$$

and $A \cap B=\varnothing$, is said to be super-additive. A capacity $\mu$ is said to be sub-additive when $\mu(A \cup$ $B) \leqslant \mu(A)+\mu(B), \forall A, B \subseteq X$ and $A \cap B=\varnothing$. Also, a super-additive capacity $\mu$ defined on $X$ is $n$ monotone, where $n>0$ and $n \in \mathrm{N}$, if and only if for any set $\mathcal{A}=\left\{A_{i} \mid 0 \leqslant i \leqslant n, A_{i} \subset X\right\}$, we have

$$
\mu\left(\bigcup_{A_{i} \in \mathcal{A}} A_{i}\right) \geqslant \sum_{I \subseteq \mathcal{A}}(-1)^{|I|+1} \mu\left(\bigcap_{A_{i} \in I} A_{i}\right) .
$$

An $n$-monotone capacity is also called a Choquet capacity of order $n$.

Definition 2 (see Section 2 of Ref. 1) Given $X=$ $\left\{x_{1}, x_{2}, \ldots, x_{n}\right\}$ as the set of realizations of uncertainty information and a family of intervals $L=$ $\left\{\left[l_{i}, u_{i}\right], i=1,2, \ldots, n \mid 0 \leqslant l_{i} \leqslant u_{i} \leqslant 1\right\}$, we define the set $\mathcal{P}^{L}$ of probability distributions on $X$ as

$$
\begin{gathered}
\mathcal{P}^{L}=\left\{p \mid l_{i} \leqslant p\left(\left\{x_{i}\right\}\right) \leqslant u_{i}, \sum_{i=1}^{n} p\left(\left\{x_{i}\right\}\right)=1,\right. \\
\forall i=1,2, \ldots, n\},
\end{gathered}
$$

where $p\left(\left\{x_{i}\right\}\right)$ is a probability density of $\left\{x_{i}\right\}$.

The set $L$ is called a set of probability intervals, or a probability interval, in short. The set $\mathcal{P}^{L}$ is the set of all possible probabilities associated with $L$.

Definition 3 (see Section 2 of Ref. 1) A probability interval $L=\left\{\left[l_{i}, u_{i}\right] \subseteq[0,1], i=1,2, \ldots, n\right\}$ such that

$$
\sum_{i=1}^{n} l_{i} \leqslant 1 \leqslant \sum_{i=1}^{n} u_{i}
$$

is called proper probability interval, which means that its associated set $\mathcal{P}^{L}$ is nonempty.

It is clear that $\mathcal{P}^{L}$ would be empty if $\sum_{i=1}^{n} l_{i}>$ 1 or $\sum_{i=1}^{n} u_{i}<1$, since any probability function $p\left(\left\{x_{i}\right\}\right)$ in $\mathcal{P}^{L}$ is defined between $l_{i}$ and $u_{i}$. For a nonempty bounded feasible region $\mathcal{P}^{L}$, we always have $\min _{p \in \mathcal{P}^{L}} p(A)$ and $\max _{p \in \mathcal{P}^{L}} p(A)$; i.e.,

$$
\min _{p \in \mathcal{P}^{L}} p(A) \leqslant p(A) \leqslant \max _{p \in \mathcal{P}^{L}} p(A), \forall A \in P(X),
$$

where $P(X)$ is the power set of the set $X$. This means that for any given subsets $A$ and $A^{\mathrm{c}}$ of $X$, where $A \cap A^{\mathrm{c}}=\varnothing$, there exist probabilities $p_{A}$ and
$q_{A^{\mathrm{c}}} \in \mathcal{P}^{L}$ such that $p_{A}(A)=\min _{p \in \mathcal{P}^{L}} p(A)$ and $q_{A^{\mathrm{c}}}\left(A^{\mathrm{c}}\right)=\min _{p \in \mathcal{P}^{L}} p\left(A^{\mathrm{c}}\right)$. Hence, $1=p_{A}(A)+$ $p_{A}\left(A^{\mathrm{c}}\right) \geqslant p_{A}(A)+q_{A^{\mathrm{c}}}\left(A^{\mathrm{c}}\right)$. The inequality $1 \geqslant$ $p_{A}(A)+q_{A^{\mathrm{c}}}\left(A^{\mathrm{c}}\right)$ is viewed as the avoid sure loss property proposed by Walley ${ }^{8}$. Hence the condition (2) is a requirement of this property. We define

$$
l(A)=\min _{p \in \mathcal{P}^{L}} p(A) \text { and }
$$

$$
u(A)=\max _{p \in \mathcal{P}^{L}} p(A), \forall A \in P(X) .
$$

Functions $l$ and $u$ are not probabilities in general, since $l(A)+l\left(A^{\mathrm{c}}\right)$ may be less than 1 and $u(A)+$ $u\left(A^{\mathrm{c}}\right)$ could be greater than 1.

Definition 4 (see Section 2 of Ref. 1) A proper probability interval $L=\left\{\left[l_{i}, u_{i}\right], i=1, \ldots, n\right\}$ is called reachable if

$$
\sum_{j \neq i} l_{j}+u_{i} \leqslant 1 \text { and } \sum_{j \neq i} u_{j}+l_{i} \geqslant 1, \forall i .
$$

The definition of a reachable probability interval implies that $\exists p_{i}$ and $p^{i} \in \mathcal{P}^{L}$ such that $l_{i}=p_{i}\left(\left\{x_{i}\right\}\right)$ and $u_{i}=p^{i}\left(\left\{x_{i}\right\}\right)$, for all $i$. Normally, $l\left(\left\{x_{i}\right\}\right) \geqslant l_{i}$ and $u\left(\left\{x_{i}\right\}\right) \leqslant u_{i}$ as defined in (3). However, when $L$ is a set of reachable probability interval, it was proved in Ref. 1 that: (i) $l\left(\left\{x_{i}\right\}\right)=l_{i}$ and $u\left(\left\{x_{i}\right\}\right)=u_{i}$ for all $i$; (ii) $l(A)$ and $u(A)$ can be calculated using the values $l_{i}$ and $u_{i}$ as

$$
\begin{aligned}
& l(A)=\max \left(\sum_{x_{i} \in A} l_{i}, 1-\sum_{x_{i} \in A^{\mathrm{c}}} u_{i}\right), \\
& u(A)=\min \left(\sum_{x_{i} \in A} u_{i}, 1-\sum_{x_{i} \in A^{\mathrm{c}}} l_{i}\right),
\end{aligned}
$$

$\forall A \in P(X)$. The $l$ and $u$ functions in (5) and (6) were proven in Ref. 1 to be Choquet capacity of order 2, which resembles 'coherence' in Ref. 8. This implies that the condition (4) is required for 'coherence constraints'- super additive and sub additive constraints. 'Avoid sure loss' and 'coherence' concepts are fully explained in Chapters 1 and 2 of Ref. 8.

Hence, if we have a reachable probability interval, then

$$
\begin{array}{r}
\left\{p \mid l_{i} \leqslant p\left(\left\{x_{i}\right\}\right) \leqslant u_{i}, \sum_{i=1}^{n} p\left(\left\{x_{i}\right\}\right)=1, \forall i\right\} \\
=\{p \mid l(A) \leqslant p(A) \leqslant u(A), \forall A \in P(X)\}
\end{array}
$$

where $l(A)$ and $u(A)$ are defined by (5) and (6). We use the notation $\mathcal{P}_{L}$ to represent the set $\{p \mid l(A) \leqslant$ 
$p(A) \leqslant u(A), \forall A \in P(X)\}$. Note that the set $\mathcal{P}^{L}$ in (1) was written to represent the set of probabilities of an arbitrary probability interval, while the set $\mathcal{P}_{L}$ is more specific for any reachable probability interval. In $\mathcal{P}_{L}$, the largest lower bound and the smallest upper bound of $p(A)$ are clearly given by $l(A)$ and $u(A)$, for each $A \in P(X)$.

Next, we give the definitions of a $\sigma$-algebra, a measurable space, a measurable mapping, and a probability space. They are used in the definition of a random set. Readers can also find the definitions of these terms in many standard probability measure textbooks, e.g., Ref. 14. Then, we discuss belief and plausibility measures.

Definition 5 (see Chapters 1 and 2 of Ref. 14) Let $\Omega$ be a nonempty set. A $\sigma$-algebra on $\Omega$, denoted by $\sigma_{\Omega}$, is a family of subsets of $\Omega$ that satisfies the properties: (i) $\varnothing \in \sigma_{\Omega}$; (ii) $B \in \sigma_{\Omega} \Rightarrow B^{\mathrm{c}} \in \sigma_{\Omega}$; (iii) $B_{i} \in \sigma_{\Omega}$, for any countable family $\left\{B_{i}\right\}_{i=1}^{\infty}$ of subsets of $\sigma_{\Omega} \Rightarrow$ $\cup_{i} B_{i} \in \sigma_{\Omega}$. A pair $\left(\Omega, \sigma_{\Omega}\right)$ is called a measurable space.

Definition 6 (see Chapters 1 and 2 of Ref. 14) Let $\left(\Omega, \sigma_{\Omega}\right)$ be a measurable space. By a measure on this space, we mean a function $\mu: \sigma_{\Omega} \rightarrow[0, \infty]$ with the properties: (i) $\mu(\varnothing)=0$; (ii) if $B_{i} \in \sigma_{\Omega}, \forall i=$ $1,2, \ldots$, are disjoint, then

$$
\mu\left(\bigcup_{i=1}^{\infty} B_{i}\right)=\sum_{i=1}^{\infty} \mu\left(B_{i}\right) .
$$

We refer to the triple $\left(\Omega, \sigma_{\Omega}, \mu\right)$ as a measure space. If $\mu(\Omega)=1$, we refer to it as a probability space and write it as $\left(\Omega, \sigma_{\Omega}, P r_{\Omega}\right)$, where $\operatorname{Pr}_{\Omega}$ is a probability measure.

Definition 7 (see Chapters 1 and 2 of Ref. 14) Let $\left(\Omega, \sigma_{\Omega}\right)$ and $\left(X, \sigma_{X}\right)$ be measurable spaces. A function $f: \Omega \rightarrow X$ is said to be a $\left(\sigma_{\Omega}, \sigma_{X}\right)$-measurable mapping if for each $A \in \sigma_{X}$,

$$
f^{-1}(A)=\{\omega \in \Omega: f(\omega) \in A\} \in \sigma_{\Omega} .
$$

Definition 8 (see Chapter 5 of Ref. 15) Let $\left(\Omega, \sigma_{\Omega}, P r_{\Omega}\right)$ be a probability space and $\left(\mathcal{F}, \sigma_{\mathcal{F}}\right)$ be a measurable space, where $\mathcal{F} \subseteq \sigma_{X}, X \neq \varnothing$, and $\left(X, \sigma_{X}\right)$ is a measurable space. A random set $\Gamma$ is a $\left(\sigma_{\Omega}, \sigma_{\mathcal{F}}\right)$-measurable mapping

$$
\begin{aligned}
\Gamma: \Omega & \rightarrow \mathcal{F} \\
\omega & \mapsto \Gamma(\omega) .
\end{aligned}
$$

A random set can be represented as a basic probability assignment function $m$ over $P(X)$ :

$$
m(E)=p(\{\omega, \Gamma(\omega)=E\}), \forall E \in P(X),
$$

such that $\sum_{E \in P(X)} m(E)=1$ and $m(\varnothing)=0$ when $X$ is finite. A set $E \in P(X)$, where $m(E)>0$ is called a focal element of $m$. We denote $F$ as the set of all focal elements. Hence, we could define a random set using the ordered pair $(F, m)$. We will see that we can formulate belief and plausibility measures from a given basic assignment $m$.

Definition 9 (See Chapter 7 of Ref. 16) Let $X$ be a finite set of realizations. A belief measure is a function

$$
\text { Bel }: P(X) \rightarrow[0,1]
$$

such that $\operatorname{Bel}(\varnothing)=0, \operatorname{Bel}(X)=1$, and it contains a super-additive property for all possible families of subsets of $X$, that is,

$$
\begin{gathered}
\operatorname{Bel}\left(A_{1} \cup \ldots \cup A_{n}\right) \geqslant \sum_{j} \operatorname{Bel}\left(A_{j}\right) \\
-\sum_{j<k} \operatorname{Bel}\left(A_{j} \cap A_{k}\right)+\ldots+(-1)^{n+1} \operatorname{Bel}\left(A_{1} \cap \ldots \cap A_{n}\right)
\end{gathered}
$$

where $A_{1}, A_{2}, \ldots, A_{n} \subseteq X$. This property of belief measures is a weaker version of the additive property of probability measures. Thus for any $A, A^{\mathrm{c}} \subseteq X$,

$$
\operatorname{Bel}(A)+\operatorname{Bel}\left(A^{\mathrm{c}}\right) \leqslant 1 .
$$

A plausibility measure, $\mathrm{Pl}$, is defined by

$$
\operatorname{Pl}(A)=1-\operatorname{Bel}\left(A^{\mathrm{c}}\right), \forall A \in P(X) .
$$

Similarly, $\operatorname{Bel}(A)=1-\operatorname{Pl}\left(A^{\mathrm{c}}\right), \forall A \in P(X)$.

Belief and plausibility measures can be characterized by a basic probability assignment function $m$, which is defined on $P(X)$ to $[0,1]$, such that $m(\varnothing)=$ 0 and $\sum_{E \in P(X)} m(E)=1$. For any $A \in P(X)$, it can be seen in Ref. 4 that

$$
\begin{aligned}
\operatorname{Bel}(A) & =\sum_{E, E \subseteq A} m(E), \text { and } \\
\operatorname{Pl}(A) & =1-\operatorname{Bel}\left(A^{\mathrm{c}}\right)=\sum_{E, E \cap A \neq \varnothing} m(E) .
\end{aligned}
$$

Also, the Bel function is $\infty$-monotone (the proof is found in Ref. 4).

A random set induces the following set of all probabilities,

$$
\mathcal{P}_{\text {Bel }}=\{p \mid \forall A \in P(X), \operatorname{Bel}(A) \leqslant p(A) \leqslant \operatorname{Pl}(A)\} .
$$

An approximation of a belief function Bel constructed from a reachable probability interval $L$ was 
given by Lemmer and Kyburg ${ }^{10}$. Their method showed that $\operatorname{Bel}(A) \geqslant l(A)$, so we have $\mathcal{P}_{\text {Bel }} \subset \mathcal{P}_{L}$. Hence this belief function Bel is an inner approximation of the probability interval $L$. On the other hand, an outer approximation of the probability interval $L$ developed by Deneoeux ${ }^{11}$ is a belief function Bel such that $\operatorname{Bel}(A) \leqslant l(A)$. This means $\mathcal{P}_{L} \subset \mathcal{P}_{\text {Bel }}$. The aim of this paper is to find conditions on a reachable probability interval $L$ necessary so that $l(A)=\operatorname{Bel}(A)$ and $u(A)=1-\operatorname{Bel}\left(A^{\mathrm{c}}\right)=\operatorname{Pl}(A)$ for all $A \in P(X)$. If this is the case, $\mathcal{P}_{L}=\mathcal{P}_{\text {Bel }}$. This also means that we have found the conditions for probability intervals to be $\infty$-monotone. In the next section, we will discuss these conditions of a probability interval.

\section{WHEN A PROBABILITY INTERVAL IS A RANDOM SET}

We suppose that $l(A)=\operatorname{Bel}(A)$ and $u(A)=\operatorname{Pl}(A)$, $\forall A \in P(X)$ in order to verify that $\mathcal{P}_{L}=\mathcal{P}_{\text {Bel }}$. Thus we need the conditions that can check the following equations:

$$
\begin{aligned}
\operatorname{Bel}(A) & =\max \left(\sum_{x_{i} \in A} l_{i}, 1-\sum_{x_{i} \in A^{\mathrm{c}}} u_{i}\right), \text { and } \\
\operatorname{Pl}(A) & =\min \left(\sum_{x_{i} \in A} u_{i}, 1-\sum_{x_{i} \in A^{\mathrm{c}}} l_{i}\right) .
\end{aligned}
$$

However, we have $l\left(A^{\mathrm{c}}\right)+u(A)=1$ by observing the calculation of $l\left(A^{\mathrm{c}}\right)$ and $u(A)$ in (5) and (6). Then, we can compute the value of $u(A)$ as $u(A)=$ $1-l\left(A^{\mathrm{c}}\right)$. Also, since $\operatorname{Pl}(A)=1-\operatorname{Bel}\left(A^{\mathrm{c}}\right)$ is calculated in a similar way to $u(A)$, we consider only the conditions of when $l(A)$ and $\operatorname{Bel}(A)$ have the same value.

Suppose $l(A)=\operatorname{Bel}(A), \forall A \subset X$. This means that the basic assignment function $m$ needs to have a special pattern. We analyse this pattern through all sizes of the nonempty subset $A$.

When $|A|=1, A=\left\{x_{i}\right\}$. The equation $\operatorname{Bel}(A)=\sum_{E, E \subset A} m(E)$ can be written as $\operatorname{Bel}\left(\left\{x_{i}\right\}\right)=m\left(\left\{x_{i}\right\}\right)$. Since we consider only the reachable probability interval, we get $l\left(\left\{x_{i}\right\}\right)=l_{i}$ and $u\left(\left\{x_{i}\right\}\right)=u_{i}$ for all $i$. Thus we must set $m\left(\left\{x_{i}\right\}\right)=$ $l_{i}$ for all $i$, so that $\operatorname{Bel}\left(\left\{x_{i}\right\}\right)=l\left(\left\{x_{i}\right\}\right)$.

When $|A|=2, A=\left\{x_{i}, x_{j}\right\}$. We know that $\operatorname{Bel}\left(\left\{x_{i}, x_{j}\right\}\right)=m\left(\left\{x_{i}\right\}\right)+m\left(\left\{x_{j}\right\}\right)+m\left(\left\{x_{i}, x_{j}\right\}\right)$. Hence

$$
\begin{aligned}
m\left(\left\{x_{i}, x_{j}\right\}\right) & =l\left(\left\{x_{i}, x_{j}\right\}\right)-m\left(\left\{x_{i}\right\}\right)-m\left(\left\{x_{j}\right\}\right) \\
& =l\left(\left\{x_{i}, x_{j}\right\}\right)-l_{i}-l_{j} .
\end{aligned}
$$

When $|A|=3, A=\left\{x_{i}, x_{j}, x_{k}\right\}$. We know that $\operatorname{Bel}\left(\left\{x_{i}, x_{j}, x_{k}\right\}\right)=m\left(\left\{x_{i}\right\}\right)+$ $m\left(\left\{x_{j}\right\}\right)+m\left(\left\{x_{k}\right\}\right)+m\left(\left\{x_{i}, x_{j}\right\}\right)+m\left(\left\{x_{i}, x_{k}\right\}\right)+$ $m\left(\left\{x_{j}, x_{k}\right\}\right)+m\left(\left\{x_{i}, x_{j}, x_{k}\right\}\right)$. Hence

$m\left(\left\{x_{i}, x_{j}, x_{k}\right\}\right)$

$$
\begin{aligned}
&=l\left(\left\{x_{i}, x_{j}, x_{k}\right\}\right)- \sum_{i, j \in\{i, j, k\}} m\left(\left\{x_{i}, x_{j}\right\}\right) \\
&-\sum_{i \in\{i, j, k\}} m\left(\left\{x_{i}\right\}\right) \\
&=l\left(\left\{x_{i}, x_{j}, x_{k}\right\}\right)-\sum_{i, j \in\{i, j, k\}} m\left(\left\{x_{i}, x_{j}\right\}\right)-\sum_{i \in\{i, j, k\}} l_{i} .
\end{aligned}
$$

By induction, we can compute $m(A)$ when $1 \leqslant$ $|A| \leqslant n-1$ as follows:

$$
\begin{aligned}
& m\left(\{\underbrace{x_{i}, x_{j}, \ldots, x_{s}}_{|A|}\}\right) \\
= & l\left(\{\underbrace{x_{i}, x_{j}, \ldots, x_{s}}_{|A|}\}\right)-\sum m\left(\{\underbrace{x_{i}, x_{j}, \ldots, x_{r}}_{|A|-1}\}\right)-\ldots \\
& -\sum_{i, j \in\{i, j, \ldots, s\}} m\left(\{\underbrace{x_{i}, x_{j}}_{2}\}\right)-\sum_{i \in\{i, j, \ldots, s\}} l_{i} .
\end{aligned}
$$

When $A=X$, we can compute $m(X)$ by using the fact that for a random set, $\sum_{E \in P(X)} m(E)=1$. Hence,

$$
\begin{aligned}
m(X)=1- & \sum_{i=1}^{n} l_{i}-\sum_{x_{i}, x_{j} \in X} m\left(\left\{x_{i}, x_{j}\right\}\right) \\
& \ldots-\sum m\left(\{\underbrace{x_{i}, x_{j}, \ldots, x_{s}}_{n-1}\}\right) .
\end{aligned}
$$

We conclude the pattern of the basic assignment function $m$ that provides $l(A)=\operatorname{Bel}(A)$ for all nonempty set $A \in P(X)$, by the following system of equations:

$$
\begin{aligned}
m\left(\left\{x_{i}\right\}\right)= & l_{i}, \\
m\left(\left\{x_{i}, x_{j}\right\}\right)=l\left(\left\{x_{i}, x_{j}\right\}\right)-l_{i}-l_{j}, & (8 \mathrm{~b}) \\
m\left(\left\{x_{i}, x_{j}, x_{k}\right\}\right)=l\left(\left\{x_{i}, x_{j}, x_{k}\right\}\right) & \quad-\sum_{i, j \in\{i, j, k\}} m\left(\left\{x_{i}, x_{j}\right\}\right) \\
& -\sum_{i \in\{i, j, k\}} l_{i}, \\
& \vdots \\
m\left(\{\underbrace{x_{i}, x_{j}, \ldots, x_{s}}_{n-1}\}\right)=l\left(\left\{x_{i}, x_{j}, \ldots, x_{s}\right\}\right) & \\
& \left.-\sum m(\underbrace{x_{i}, \ldots, x_{r}}_{n-2}\}\right)
\end{aligned}
$$




$$
\begin{aligned}
& -\ldots-\sum_{i \in\{i, j, \ldots, s\}} l_{i} \\
m(X)=1 & -\sum_{i=1}^{n} l_{i} \\
& -\sum_{x_{i}, x_{j} \in X} m(\{\mathrm{~d}) \\
& -\ldots \\
& \left.-\sum m(\underbrace{\left.x_{i}, \ldots, x_{s}, x_{j}\right\}}_{n-1}\}\right) .
\end{aligned}
$$

Also, we must verify that $m(A) \geqslant 0, \forall A \in$ $P(X)$, to satisfy the random set property. This is not difficult to see when the size of $X$ is one or two, as we show below.

Case $X=\left\{x_{1}\right\}$. Since we only consider the reachable probability intervals, we have only $p\left(\left\{x_{1}\right\}\right) \in[1,1]$. Then we get $m\left(\left\{x_{1}\right\}\right)=1$.

Case $X=\left\{x_{1}, x_{2}\right\}$. Let $p\left(\left\{x_{1}\right\}\right) \in\left[l_{1}, u_{1}\right]$ and $p\left(\left\{x_{2}\right\}\right) \in\left[l_{2}, u_{2}\right]$ of a reachable probability interval. We get $l_{2}+u_{1} \leqslant 1$ and $u_{1}+l_{2} \geqslant 1$, so $l_{2}+u_{1}=1$. Similarly, we have $l_{1}+u_{2}=1$, and $l_{1}+l_{2} \leqslant 1$ since $l_{1} \leqslant u_{1}$. Let $m\left(\left\{x_{1}\right\}\right)=l_{1}$ and $m\left(\left\{x_{2}\right\}\right)=l_{2}$. So $m(X)=m\left\{x_{1}, x_{2}\right\}=1-l_{1}-l_{2} \geqslant 0$.

However, it may turn out that $m(A)<0$ using the system of (8) for a given probability interval that has $|X| \geqslant 3$. Hence we need to find the conditions of a probability interval to make sure that $m(A) \geqslant 0$. These conditions are stated in Theorem 1 but we need Lemma 1 in order to prove the theorem.

Lemma 1 Let $X=\left\{x_{1}, x_{2}, \ldots, x_{n}\right\}$ with $n \geqslant$ 3. For a given reachable probability interval $L=$ $\left\{\left[l_{i}, u_{i}\right], i=1,2, \ldots, n \mid 0 \leqslant l_{i} \leqslant u_{i} \leqslant 1\right\}$, if $m\left(\left\{x_{i}\right\}\right)=l_{i}$ and there exists an index $i$ such that $\sum_{j \neq i} l_{j}+u_{i}=1$, then $m(A)=0 \forall A \in P\left(X \backslash\left\{x_{i}\right\}\right)$ with $|A| \geqslant 2$.

Proof: Without loss of generality, we assume $i=1$. Hence $l_{2}+l_{3}+\ldots+l_{n}+u_{1}=1$. We will prove the lemma by mathematical induction on the size of $A$.

Basic step. When $|A|=2$. Let $A=\left\{x_{j_{1}}, x_{j_{2}}\right\}$, $j_{1}<j_{2}$. By assumption we get

$$
\begin{aligned}
l_{j_{1}}+l_{j_{2}}=1 & -l_{2}-l_{3}-\ldots-l_{j_{1}-1}-l_{j_{1}+1} \\
& \quad \ldots-l_{j_{2}-1}-l_{j_{2}+1}-l_{n}-u_{1} \\
>1 & -u_{2}-u_{3}-\ldots-u_{j_{1}-1}-u_{j_{1}+1} \\
& \quad \ldots-u_{j_{2}-1}-u_{j_{2}+1}-u_{n}-u_{1} .
\end{aligned}
$$

We get $l\left(\left\{x_{j_{1}}, x_{j_{2}}\right\}\right)=l_{j_{1}}+l_{j_{2}}$, due to (5). Also, since we want $\operatorname{Bel}\left(\left\{x_{j_{1}}, x_{j_{2}}\right\}\right)=l\left(\left\{x_{j_{1}}, x_{j_{2}}\right\}\right)$ and we know that $\operatorname{Bel}\left(\left\{x_{j_{1}}, x_{j_{2}}\right\}\right)=$ $m\left(\left\{x_{j_{1}}\right\}\right)+m\left(\left\{x_{j_{2}}\right\}\right)+m\left(\left\{x_{j_{1}}, x_{j_{2}}\right\}\right)$, we get $m\left(\left\{x_{j_{1}}, x_{j_{2}}\right\}\right)=0$.

Induction step. Let $m(A)=0, \forall A \in P(X \backslash$ $\left.\left\{x_{1}\right\}\right)$, where $|A|=k, k \leqslant n-2$. Consider $l_{2}+$ $l_{3}+\ldots+l_{n}=1-u_{1}$, we get $l\left(\left\{x_{2}, x_{3}, \ldots, x_{n}\right\}\right)=$ $l_{2}+l_{3}+\ldots+l_{n}$. From

$$
\begin{gathered}
m\left(\{\underbrace{x_{i}, x_{j}, \ldots, x_{s}}_{n-1}\}\right)=l\left(\left\{x_{i}, x_{j}, \ldots, x_{s}\right\}\right) \\
-\sum m\left(\{\underbrace{x_{i}, x_{j}, \ldots, x_{r}}_{n-2}\}\right) \\
\quad-\ldots-\sum_{i \in\{i, j, \ldots, s\}} l_{i}
\end{gathered}
$$

and $m(A)=0$ for all $A \in P\left(X \backslash\left\{x_{1}\right\}\right)$, where $|A|=k, k \leqslant n-2$, we have $m\left(\left\{x_{2}, x_{3}, \ldots, x_{n}\right\}\right)=$ $l\left(\left\{x_{2}, x_{3}, \ldots, x_{n}\right\}\right)-\sum_{i \in\{2,3, \ldots, n\}} l_{i}=0$.

By induction, we conclude that if there exists an index $i$ such that $\sum_{j \neq i} l_{j}+u_{i}=1$ then with $|A| \geqslant 2$, $m(A)=0, \forall A \in P\left(X \backslash\left\{x_{i}\right\}\right)$.

Remark 1 When $|X| \geqslant 3$, if we have $\sum_{j \neq i} l_{j}+u_{i}<$ $1, \forall i$, we may have $m(A)<0$ for some subset $A$ of $X$, as shown in the following example in the case when $|X|=3$.

Example 1 Consider $X=\left\{x_{1}, x_{2}, x_{3}\right\}$ with a reachable probability interval $L=\left\{\left[l_{i}, u_{i}\right] \mid 0 \leqslant l_{i} \leqslant u_{i} \leqslant\right.$ $1, i=1,2,3\}$ where $\sum_{j \neq i} l_{j}+u_{i}<1, \forall i$. Let $m\left(\left\{x_{i}\right\}\right)=l_{i}$. Since $l_{i}+l_{j}<1-u_{k}$, we then get $l\left(\left\{x_{i}, x_{j}\right\}\right)=1-u_{k}$ from (5). Hence

$$
\begin{aligned}
m\left(\left\{x_{i}, x_{j}\right\}\right) & =l\left(\left\{x_{i}, x_{j}\right\}\right)-l_{i}-l_{j} \\
& =1-u_{k}-l_{i}-l_{j} \\
& =1-(\underbrace{u_{k}+l_{i}+l_{j}}_{<1})
\end{aligned}
$$$$
\geqslant 0 \text {. }
$$

Now, let us consider $m(X)$.

$$
\begin{aligned}
m(X)= & 1-\sum_{i=1}^{3} m\left(\left\{x_{i}\right\}\right)-\sum_{i \neq j} m\left(\left\{x_{i}, x_{j}\right\}\right) \\
= & 1-l_{1}-l_{2}-l_{3}-\left(1-u_{1}-l_{2}-l_{3}\right. \\
& \left.\quad+1-u_{2}-l_{1}-l_{3}+1-u_{3}-l_{1}-l_{2}\right) \\
= & l_{1}+l_{2}+l_{3}+u_{1}+u_{2}+u_{3}-2 \\
= & \sum_{i=1}^{3} l_{i}+\sum_{i=1}^{3} u_{i}-2 .
\end{aligned}
$$


Since we do not know the value of $\sum_{i=1}^{3} l_{i}+\sum_{i=1}^{3} u_{i}$, we could not conclude that $m(X)$ is nonnegative.

Theorem 1 Let $X=\left\{x_{1}, x_{2}, \ldots, x_{n}\right\}, n \geqslant 3$. For $a$ given reachable probability interval $L=\left\{\left[l_{i}, u_{i}\right] \mid 0 \leqslant\right.$ $\left.l_{i} \leqslant u_{i} \leqslant 1, i=1,2, \ldots, n\right\}$, if there are at most two indices, say $i_{1}, i_{2}$, such that $\sum_{j \neq i_{1}} l_{j}+u_{i_{1}}<1$ and $\sum_{j \neq i_{2}} l_{j}+u_{i_{2}}<1$, then we can construct the unique random set that has the same information as the probability interval $L$, i.e., $\mathcal{P}_{\mathrm{Bel}}=\mathcal{P}_{L}$, which means $\operatorname{Bel}(A)=l(A)$ and $\operatorname{Pl}(A)=u(A), \forall A \in$ $P(X)$.

Proof: If we have an extra index called $i_{3}$, where $i_{3} \neq$ $i_{1}$ or $i_{2}$, such that $\sum_{j \neq i_{3}} l_{j}+u_{i_{3}}<1$, then we cannot construct the random set that has the same information as the probability interval $L$, in general, as shown in Remark 1 and Example 1. Thus we organize our proof into the following three cases.

Case 1. There are two indices, say $i_{1}, i_{2}$, such that $\sum_{j \neq i_{1}} l_{j}+u_{i_{1}}<1$ and $\sum_{j \neq i_{2}} l_{j}+u_{i_{2}}<1$. Without loss of generality, let $i_{1}=1$ and $i_{2}=2$.

(i) When $n=3$. Since $\sum_{j \neq 1} l_{j}+u_{1}<1$ and $\sum_{j \neq 2} l_{j}+u_{2}<1$, we get $\sum_{j \neq 3}^{j \neq 1} l_{j}+u_{3}=1$ by the property of reachable probability intervals. Let $m\left(\left\{x_{i}\right\}\right)=l_{i}$. By Lemma 1 , we get $m\left(\left\{x_{1}, x_{2}\right\}\right)=$ 0 . Next, we will show that $m\left(\left\{x_{1}, x_{3}\right\}\right), m\left(\left\{x_{2}, x_{3}\right\}\right)$ and $m(X)$ have nonnegative values, to complete this part. Since $l_{1}+l_{3}<1-u_{2}$ and $l_{2}+l_{3}<1-u_{1}$, we then get $l\left(\left\{x_{1}, x_{3}\right\}\right)=1-u_{2}$ and $l\left(\left\{x_{2}, x_{3}\right\}\right)=$ $1-u_{1}$, using (5). Hence,

$$
\begin{aligned}
m\left(\left\{x_{1}, x_{3}\right\}\right) & =l\left(\left\{x_{1}, x_{3}\right\}\right)-l_{1}-l_{3} \\
& =1-u_{2}-l_{1}-l_{3} \\
& =1-(\underbrace{u_{2}+l_{1}+l_{3}}_{<1}) \geqslant 0 . \\
m\left(\left\{x_{2}, x_{3}\right\}\right) & =l\left(\left\{x_{2}, x_{3}\right\}\right)-l_{2}-l_{3} \\
= & 1-u_{1}-l_{2}-l_{3} \geqslant 0 . \\
m(X)= & 1-\sum m\left(\left\{x_{i}\right\}\right)-\sum m\left(\left\{x_{i}, x_{j}\right\}\right) \\
= & 1-l_{1}-l_{2}-l_{3}-\left(1-u_{2}-l_{1}\right. \\
& \left.-l_{3}+1-u_{1}-l_{2}-l_{3}\right) \\
& =\underbrace{u_{2}+u_{1}+l_{3}}_{\geqslant 1}-1 \geqslant 0 .
\end{aligned}
$$

(ii) When $n \geqslant 4$. We have $i=1,2$ such that $\sum_{j \neq i} l_{j}+u_{i}<1$. Hence

$$
\begin{aligned}
& l_{2}+l_{3}+\ldots+l_{n}<1-u_{1} \\
& l_{1}+l_{3}+\ldots+l_{n}<1-u_{2} .
\end{aligned}
$$

Hence $l\left(\left\{x_{2}, x_{3}, \ldots, x_{n}\right\}\right)=1-u_{1}$ and $l\left(\left\{x_{1}, x_{3}, \ldots, x_{n}\right\}\right)=1-u_{2}$ by (5). For $i \neq 1,2$, we get $\sum_{j \neq i} l_{j}+u_{i}=1$. Set $m\left(\left\{x_{i}\right\}\right)=l_{i}$, we obtain $m(A)=0, \forall A \in P\left(X \backslash\left\{x_{i}\right\}\right) i \neq 1,2$, by Lemma 1. Hence we have $m(A)=0, \forall A \in P(X) \backslash$ $\left\{X,\left\{x_{3}, \ldots, x_{n}\right\},\left\{x_{1}, x_{3}, \ldots, x_{n}\right\},\left\{x_{2}, x_{3}, \ldots, x_{n}\right\}\right\}$ Hence, we must find the value of $m\left(\left\{x_{3}, \ldots, x_{n}\right\}\right)$, $m\left(\left\{x_{1}, x_{3}, \ldots, x_{n}\right\}\right), \quad m\left(\left\{x_{2}, x_{3}, \ldots, x_{n}\right\}\right) \quad$ and $m(X)$. From (8), we obtain

$$
\begin{aligned}
l_{3}+l_{4}+\ldots+l_{n}+m\left(\left\{x_{3}, \ldots, x_{n}\right\}\right) & \\
& =l\left(\left\{x_{3}, x_{4}, \ldots, x_{n}\right\}\right)
\end{aligned}
$$

$$
\begin{aligned}
l_{1}+l_{3}+l_{4}+ & \ldots+l_{n}+m\left(\left\{x_{3}, \ldots, x_{n}\right\}\right) \\
& +m\left(\left\{x_{1}, x_{3}, \ldots, x_{n}\right\}\right)=1-u_{2} \\
l_{2}+l_{3}+l_{4}+ & \ldots+l_{n}+m\left(\left\{x_{3}, \ldots, x_{n}\right\}\right) \\
& +m\left(\left\{x_{2}, x_{3}, \ldots, x_{n}\right\}\right)=1-u_{1}
\end{aligned}
$$

$$
\begin{aligned}
\sum_{i=1}^{n} l_{i}+ & m\left(\left\{x_{3}, \ldots, x_{n}\right\}\right)+m\left(\left\{x_{1}, x_{3}, \ldots, x_{n}\right\}\right) \\
& +m\left(\left\{x_{2}, x_{3}, \ldots, x_{n}\right\}\right)+m(X)=1 .
\end{aligned}
$$

Thus we can write this system of equations in the form of matrix notation by using $m(X)$, $m\left(\left\{x_{3}, \ldots, x_{n}\right\}\right), m\left(\left\{x_{1}, x_{3}, \ldots, x_{n}\right\}\right), \quad$ and $m\left(\left\{x_{2}, x_{3}, \ldots, x_{n}\right\}\right)$ as unknown variables.

$$
\begin{gathered}
{\left[\begin{array}{llll}
1 & 0 & 0 & 0 \\
1 & 1 & 0 & 0 \\
1 & 0 & 1 & 0 \\
1 & 1 & 1 & 1
\end{array}\right]\left[\begin{array}{l}
m\left(\left\{x_{3}, \ldots, x_{n}\right\}\right) \\
m\left(\left\{x_{1}, x_{3}, \ldots, x_{n}\right\}\right) \\
m\left(\left\{x_{2}, x_{3}, \ldots, x_{n}\right\}\right) \\
m(X)
\end{array}\right]} \\
=\left[\begin{array}{l}
l\left(\left\{x_{3}, \ldots, x_{n}\right\}\right)-l_{3}-l_{4}-\ldots-l_{n} \\
1-u_{2}-l_{1}-l_{3}-l_{4}-\ldots-l_{n} \\
1-u_{1}-l_{2}-l_{3}-l_{4}-\ldots-l_{n} \\
1-l_{1}-l_{2}-l_{3}-\ldots-l_{n}
\end{array}\right],
\end{gathered}
$$

which means

$$
\begin{aligned}
& {\left[\begin{array}{l}
m\left(\left\{x_{3}, \ldots, x_{n}\right\}\right) \\
m\left(\left\{x_{1}, x_{3}, \ldots, x_{n}\right\}\right) \\
m\left(\left\{x_{2}, x_{3}, \ldots, x_{n}\right\}\right) \\
m(X)
\end{array}\right]} \\
& =\left[\begin{array}{l}
l\left(\left\{x_{3}, \ldots, x_{n}\right\}\right)-l_{3}-l_{4}-\ldots-l_{n} \\
-l\left(\left\{x_{3}, \ldots, x_{n}\right\}\right)+1-u_{2}-l_{1} \\
-l\left(\left\{x_{3}, \ldots, x_{n}\right\}\right)+1-u_{1}-l_{2} \\
l\left(\left\{x_{3}, \ldots, x_{n}\right\}\right)-1+u_{1}+u_{2}
\end{array}\right] .
\end{aligned}
$$

Since $l(A)=\max \left(\sum_{x_{i} \in A} l_{i}, 1-\sum_{x_{i} \in A^{\mathrm{c}}} u_{i}\right)$, we have

$$
\begin{aligned}
& m\left(\left\{x_{3}, \ldots, x_{n}\right\}\right) \\
& \quad=l\left(\left\{x_{3}, \ldots, x_{n}\right\}\right)-l_{3}-l_{4}-\ldots-l_{n} \geqslant 0
\end{aligned}
$$


and

$$
\begin{aligned}
m(X) & =l\left(\left\{x_{3}, \ldots, x_{n}\right\}\right)-1+u_{1}+u_{2} \\
& \geqslant 1-u_{1}-u_{2}-1+u_{1}+u_{2}=0 .
\end{aligned}
$$

We must show that $m\left(\left\{x_{1}, x_{3}, \ldots, x_{n}\right\}\right) \geqslant 0$ and $m\left(\left\{x_{2}, x_{3}, \ldots, x_{n}\right\}\right) \geqslant 0$ to complete the proof of this case. Consider when $l\left(\left\{x_{3}, \ldots, x_{n}\right\}\right)=l_{3}+l_{4}+$ $\ldots+l_{n}$, so

$$
\begin{aligned}
& m\left(\left\{x_{1}, x_{3}, \ldots, x_{n}\right\}\right) \\
& =-l\left(\left\{x_{3}, \ldots, x_{n}\right\}\right)+1-u_{1}-l_{2} \\
& =-(\underbrace{l_{2}+l_{3}+l_{4}+\ldots+l_{n}+u_{1}}_{<1})+1 \geqslant 0 .
\end{aligned}
$$

When $l\left(\left\{x_{3}, \ldots, x_{n}\right\}\right)=1-u_{1}-u_{2}$, then

$$
\begin{aligned}
& m\left(\left\{x_{1}, x_{3}, \ldots, x_{n}\right\}\right) \\
& =-l\left(\left\{x_{3}, \ldots, x_{n}\right\}\right)+1-u_{1}-l_{2} \\
& =-1+u_{1}+u_{2}+1-u_{1}-l_{2}=u_{2}-l_{2} \geqslant 0 .
\end{aligned}
$$

Hence $-l\left(\left\{x_{3}, \ldots, x_{n}\right\}\right)+1-u_{2}-l_{1} \geqslant 0$, and $m\left(\left\{x_{1}, x_{3}, \ldots, x_{n}\right\}\right) \geqslant 0$. We can obtain $m\left(\left\{x_{2}, x_{3}, \ldots, x_{n}\right\}\right) \geqslant 0$ in a similar fashion.

Case 2. There is only one index, say $i_{1}$, such that $\sum_{j \neq i_{1}} l_{j}+u_{i_{1}}<1$. Without loss of generality, let $i_{1}=1$. Then we have $l_{2}+l_{3}+\ldots+l_{n}<1-u_{1}$. Hence $l\left(\left\{x_{2}, x_{3}, \ldots, x_{n}\right\}\right)=1-u_{1}$. For $i \neq 1$, we get $\sum_{j \neq i} l_{j}+u_{i}=1$. Let $m\left(\left\{x_{i}\right\}\right)=l_{i}$, we obtain $m(A)=0, \forall A \in P\left(X \backslash\left\{x_{i}\right\}\right) \forall i \neq 1$, by Lemma 1 . Hence we have

$$
m(A)=0, \forall A \in P(X) \backslash\left\{\left\{x_{2}, x_{3}, \ldots, x_{n}\right\}, X\right\} .
$$

Hence we must show that $m\left(\left\{x_{2}, x_{3}, \ldots, x_{n}\right\}\right) \geqslant 0$ and $m(X) \geqslant 0$ to complete the proof. Eq. (8d) provides

$$
\begin{array}{r}
m\left(\{\underbrace{x_{i}, x_{j}, \ldots, x_{s}}_{n-1}\}\right) \\
=l\left(\left\{x_{i}, x_{j}, \ldots, x_{s}\right\}\right)-\sum m\left(\{\underbrace{x_{i}, x_{j}, \ldots, x_{r}}_{n-2}\}\right) \\
-\ldots-\sum_{i \in\{i, j, \ldots, s\}} l_{i},
\end{array}
$$

which means

$$
\begin{aligned}
& m\left(\left\{x_{2}, x_{3}, \ldots, x_{n}\right\}\right) \\
& =l\left(\left\{x_{2}, x_{3}, \ldots, x_{n}\right\}\right)-\sum_{i \in\{2,3, \ldots, n\}} l_{i} \\
& =1-u_{1}-l_{2}-l_{3}-\ldots-l_{n} \\
& =1-(\underbrace{u_{1}+l_{2}+l_{3}+\ldots+l_{n}}_{<1}) \geqslant 0 .
\end{aligned}
$$

From the last equation of (8), we also have

$$
\begin{aligned}
m(X)=1- & \sum l_{i}-\sum m\left(\left\{x_{i}, x_{j}\right\}\right) \\
& -\ldots-\sum m\left(\{\underbrace{x_{i}, x_{j}, \ldots, x_{s}}_{n-1}\}\right) .
\end{aligned}
$$

Hence

$$
\begin{aligned}
m(X) & =1-\sum l_{i}-m\left(\left\{x_{2}, x_{3}, \ldots, x_{n}\right\}\right) \\
& =1-\sum l_{i}-\left(1-u_{1}-l_{2}-l_{3}-\ldots-l_{n}\right) \\
& =u_{1}-l_{1} \geqslant 0 .
\end{aligned}
$$

Case 3. There is no index $i$ such that

$$
\sum_{j \neq i} l_{j}+u_{i}<1 .
$$

Hence we get $\sum_{j \neq i} l_{j}+u_{i}=1, \forall i$. Let $m\left(\left\{x_{i}\right\}\right)=$ $l_{i}$, we obtain $m(A)=0, \forall A \in P\left(X \backslash\left\{x_{i}\right\}\right) \forall i$, by Lemma 1. Hence we have $m(A)=0, \forall A \in P(X) \backslash$ $X$, and $m(X)=1-\sum l_{i} \geqslant 0$.

From all of these cases, we conclude that when there are at most two indices, say $i_{1}, i_{2}$, such that $\sum_{j \neq i_{1}} l_{j}+u_{i_{1}}<1$ and $\sum_{j \neq i_{2}} l_{j}+u_{i_{2}}<1$, we can construct the unique random set that has the same information such that $\mathcal{P}_{\text {Bel }}=\mathcal{P}_{L}$, i.e., $\operatorname{Bel}(A)=l(A)$ and $\operatorname{Pl}(A)=u(A), \forall A \in P(X)$, by defining $m$ according to (8).

In the next section, we give an example of a situation where we have probability interval information and we do not want the other persons to have a clear view of our information, since occasionally this type of situation may yield a higher benefit/profit. We then represent the information by using a random set instead of a probability interval if our probability interval satisfies the conditions in Theorem 1.

\section{EXAMPLE}

In a horse-racing field, suppose that an on-looker pays $\$ 10$ to bet on a particular horse. If that horse wins, they will receive a return of $\$ 20$. Suppose further that a bettor in the horse-racing field has a probability interval information about four exceptional horses, $A$, $B, C$ and $D$, as follows.

$$
\begin{aligned}
& p(\{A \text { will be the winner }\}) \in\left[\frac{1}{4}, \frac{1}{2}\right], \\
& p(\{B \text { will be the winner }\}) \in\left[\frac{1}{4}, \frac{7}{16}\right], \\
& p(\{C \text { will be the winner }\}) \in\left[\frac{1}{8}, \frac{3}{8}\right], \\
& p(\{D \text { will be the winner }\}) \in\left[\frac{1}{8}, \frac{5}{16}\right] .
\end{aligned}
$$


Let $X$ be the set of the exceptional horses, $X=$ $\{A, B, C, D\}$. We start by checking the reachability of this probability interval. Since

$$
\begin{gathered}
l_{B}+l_{C}+l_{D}+u_{A}=1, l_{A}+l_{C}+l_{D}+u_{B}=\frac{15}{16}, \\
l_{A}+l_{B}+l_{D}+u_{C}=1, l_{A}+l_{B}+l_{C}+u_{D}=\frac{15}{16}, \\
l_{1}+u_{2}+u_{3}+u_{4}=\frac{11}{8}, l_{2}+u_{1}+u_{3}+u_{4}=\frac{5}{4}, \\
l_{3}+u_{1}+u_{2}+u_{4}=\frac{11}{8}, l_{4}+u_{1}+u_{2}+u_{3}=\frac{23}{16},
\end{gathered}
$$

this probability interval is reachable. Also, $l_{A}+l_{C}+$ $l_{D}+u_{B}=\frac{15}{16}$ and $l_{A}+l_{B}+l_{C}+u_{D}=\frac{15}{16}$ satisfy the conditions stated in Theorem 1. Thus we can use the basic assignment function in the proof of Case 1 of Theorem 1 as follows. $m(\{A\})=\frac{1}{4}, m(\{B\})=\frac{1}{4}$, $m(\{C\})=\frac{1}{8}, m(\{D\})=\frac{1}{8}, m(\{A, C, D\})=\frac{1}{16}$, $m(\{A, B, C\})=\frac{1}{16}$, and $m(X)=\frac{1}{8}$. Hence the belief that $A$ will be the winner is $\frac{1}{4}$, which equals to the belief that $B$ will be the winner. The belief that $C$ will be the winner is $\frac{1}{8}$, which equals to the belief that $D$ will be the winner. The belief that the winner will be one of the horses $A, B$, or $C$ is $\frac{11}{16}$. The belief that the winner will be one of the horses $A, C$, or $D$, is $\frac{9}{16}$. Also, the belief that the winner is one of these four horses is equal to 1, (as every one would know), assuming that the race is among these four horses.

This random set information makes it harder for an on-looker to interpret which horse would have the best chance to win. However, if the bettor provides the above probability interval to the on-looker, they may easily guess that horse $A$ has more chance to win. Hence, the random set information would gain more benefit for the bettor.

\section{CONCLUSIONS}

We obtain the conditions for which a given probability interval can be transformed into a unique random set with the same information. This probability interval must be reachable and must have at most two indices, say $i_{1}, i_{2}$, such that $\sum_{j \neq i_{1}} l_{j}+u_{i_{1}}<1$ and $\sum_{j \neq i_{2}} l_{j}+u_{i_{2}}<1$. It means that we found the conditions sufficient for a probability interval to be of $\infty$-monotone capacity. The construction of the corresponding random set is provided in the proof of Theorem 1. In a situation where a user wishes to reveal a minimum of information, it would be better if they prented the probability interval information as the unique random set when these two uncertainties refer to the same information.

Acknowledgements: The first author was a student in the Applied Mathematics and Computer Science programme at Chulalongkorn University and was supported by the Development and Promotion of Science and Technology Talents
Project (DPST). The corresponding author was supported by Grants for Development of New Faculty Staff, Chulalongkorn University, and Research Strategic Plan (A1B1), Research Funds from the Faculty of Science, Chulalongkorn University. We also thank the reviewers for their comments and suggestions.

\section{REFERENCES}

1. De Campos LM, Huete JF, Moral S (1994) Probability intervals: a tool for uncertain reasoning. IJUFKS 2 , 167-96.

2. Dempster A (1967) Upper and lower probabilites induced by a multivalued mapping. Ann Math Stat 38, 325-39.

3. Molchanov I (2005) Theory of Random Sets, Springer, London.

4. Shafer G (1976) A Mathematical Theory of Evidence, Princeton Univ Press, Princeton.

5. Destercke S, Dubois D, Chojnacki E (2008) Unifying practical uncertainty representations: I. Generalized $p$ boxes. Int J Approx Reas 49, 649-63.

6. Ferson S, Ginzburg L, Kreinovich V, Myers D, Sentz K (2003) Constructing probability boxes and DempsterShafer structures. Technical report, Sandia National Laboratories.

7. Neumaier A (2004) Clouds, fuzzy sets and probability intervals. Reliable Comput 10, 249-72.

8. Walley P (1991) Statistical Reasoning with Imprecise Probabilites, Chapman \& Hall, New York.

9. Zadeh L (1978) Fuzzy sets as a basis for a theory of possibility. Fuzzy Set Syst 1, 3-28.

10. Lemmer JF, Kyburg HE (1991) Conditions for the existence of belief functions corresponding to interval of belief. In: Proceedings of the 9th National Conference on A.I., Anaheim, California, pp 488-93.

11. Denoeux T (2006) Constructing belief functions from sample data using multinomial confidence regions. Int J Approx Reas 42, 228-52.

12. Thipwiwatpotjana P, Lodwick W (2011) The relationship between a probability interval and a random set. In: Proceedings of Annual Meeting of the North American Fuzzy Information Processing Society (NAFIPS), IEEE, El Paso, Texas, pp 1-5.

13. Thipwiwatpotjana P, Lodwick W (2013) A relationship between probability interval and random sets and its application to linear optimization with uncertainties. Fuzzy Sets Syst (in press).

14. Roussas G (2004) An Introduction to MeasureTheoretic Probability, Elsevier Academic Press, Burlington, Massachusetts.

15. Nguyen HT (2006) An Introduction to Random Sets, Chapman and Hall/CRC, Boca Raton, Florida.

16. Klir G, Yuan B (1995) Fuzzy Sets and Fuzzy Logic: Theory and Applications, Prentice Hall, New Jersey. 